\title{
The ATP-dependent PIM1 protease is required for the expression of intron-containing genes in mitochondria
}

\author{
Luc van Dyck, Walter Neupert, and Thomas Langer ${ }^{1}$ \\ Institut für Physiologische Chemie der Universität M ünchen, 80336 M ünchen, Germany
}

\begin{abstract}
The ATP-dependent PIM1 protease, a Lon-like protease localized in the mitochondrial matrix, is required for mitochondrial genome integrity in yeast. Cells lacking PIM1 accumulate lesions in the mitochondrial DNA (mtDNA) and therefore lose respiratory competence. The identification of a multicopy suppressor, which stabilizes mtDNA in the absence of PIM1, enabled us to characterize novel functions of PIM1 protease during mitochondrial biogenesis. The synthesis of mitochondrially encoded cytochrome c oxidase subunit I (Coxl) and cytochrome b (Cob) is impaired in pim 1 mutants containing mtDNA. PIM 1-mediated proteolysis is required for the translation of mature COXI mRNA. Moreover, deficiencies in the splicing of $\mathrm{COXI}$ and $\mathrm{COB}$ transcripts, which appear to be restricted to introns encoding mRNA maturases, were observed in cells lacking the PIM 1 gene. Transcripts of COXI and С OB genes harboring multiple introns are degraded in the absence of PIM1. These results establish multiple, essential functions of the ATP-dependent PIM1 protease during mitochondrial gene expression.
\end{abstract}

[Key Words: Mitochondria; ATP-dependent proteolysis; PIM 1 protease; translation; RN A processing; RN A stability; cytochrome c oxidase; cytochrome b]

Received N ovember 25, 1997; revised version accepted M arch 11, 1998.

Many cellular processes are under the control of ATPdependent proteases that ensure cellular homeostasis and allow the adaptation to changes in environmental conditions. In eukaryotic cells, the 265 proteasome, a multicatalytic proteolytic complex localized in the cytosol, mediates the energy-dependent degradation of most cellular proteins (Coux et al. 1996; Hilt and Wolf 1996; Baumeister and Lupas 1997). Other than the 265 proteasome, ATP-dependent proteases have only been identified in organelles of endosymbiotic origin, such as mitochondria and chloroplasts, which harbor independent proteolytic systems (Adam 1996; Langer and N eupert 1996; Rep and Grivell 1996; Suzuki et al. 1997). The ATP-dependent proteases of mitochondria fulfill crucial functions during the biogenesis of the organelle, as they are required for the mai ntenance of the respi ratory competence in yeast. However, their physi ologi cal substrates have not been described until now.

Two ATP-dependent proteases have been identified in the mitochondrial inner membrane and were termed AAA proteases (Leonhard et al. 1996) as their subunits contain a highly conserved domain characteristic for the AAA family of AT Pases (Kunau et al. 1993; Confalonieri and Duguet 1995). Ymelp, an integral inner membrane protein facing the intermembrane space, is the solely

${ }^{1}$ Corresponding author.

E-MAIL: Langer@bio.med.uni-muenchen.de; FAX 49895996270. identified subunit of the i-AAA protease (T horsness et al. 1993). Proteolysis by Ymelp is required for the maintenance of respiratory competence of the cells at elevated temperatures and for the formation of a reticulated network of mitochondria (Thorsness et al. 1993; Campbell et al. 1994). The m-AAA protease is composed of multiple copies of $Y$ talop and $Y$ ta12p, integral inner membrane proteins that are homol ogous to $Y$ melp but expose their catalytic sites to the mitochondrial matrix (Arlt et al. 1996). Cells lacking Yta10p or Y ta12p display deficiencies in the assembly of respiratory chain complexes (Guélin et al. 1994; Tauer et al. 1994; Tzagoloff et al. 1994). Both AAA proteases mediate the degradation of nonassembled inner membrane proteins (A rlt et al . 1996; Guélin et al. 1996). How these AAA proteases are involved in the biogenesis of the respiratory chain and in the maintenance of mitochondrial morphology, however, is still unknown.

The ATP-dependent PIM 1 protease controls the selective turnover of proteins in the mitochondrial matrix space (Suzuki et al. 1994; van Dyck et al. 1994). Misfolded polypepti des are degraded by PIM 1 protease in cooperation with the mitochondrial $\mathrm{Hsp} 70$ system that stabilizes substrate polypeptides against aggregation (Wagner et al. 1994). Overexpression of PIM 1 restores the respiratory competence of $\Delta y$ ta10 $\Delta y$ tal2 mutants, sug gesting a functional overlap with the m-AAA protease (Rep et al. 1996a). Similar to the m-AAA protease, PIM 1 
protease forms an high molecular weight, presumably homo-oligomeric complex whose assembly depends on its intrinsic ATPase activity (Wagner et al. 1997). Y east cells lacking the PIMI gene lose intact mitochondrial DNA (mtDNA) (Suzuki et al. 1994; van Dyck et al. 1994). As essential components of the respiratory chain are encoded by the mitochondrial genome, piml mutants are respiratory deficient. Electron dense particles, most likely consisting of aggregated polypeptides, were observed in mitochondria of $\Delta$ piml mutants (Suzuki et al. 1994). It was therefore speculated that the loss of mtDNA in the absence of PIM 1 may be caused by the accumulation of misfolded polypeptides (Grivell 1995). Alternatively, one may envision regulatory functions of PIM 1 protease in mtDNA metabolism.

Proteins homologous to PIM 1 are present in bacteria and mitochondria of human and plant cells and comprise the family of Lon-like proteases (Gol dberg 1992; Gottesman and Maurizi 1992; Maurizi 1992). Functional conservation of Escherichia coli Lon protease with PIM 1 has recently been demonstrated in yeast (T eichmann et al. 1996). The respiratory competence of cells lacking PI M 1, that is, the integrity of $\operatorname{mtDNA}$, can be maintained by expression of E. coli Lon protease. Although complementation depended on the proteolytic activity of the Lon protease, a mutant variant with reduced enzymatic activity, Lon ${ }^{\mathrm{K} 362 \mathrm{~A}}$ protease, was able to substitute for PIM 1 protease (Teichmann et al. 1996). A pparently, a low proteolytic activity of a Lon-like protease is sufficient to maintain the respiratory competence of the cells. Substitution of Lon protease for PIM 1 was found to occur at $30^{\circ} \mathrm{C}$ but not when cells were grown at $36^{\circ} \mathrm{C}$ indicating functional differences between the proteases (Teichmann et al. 1996).

In the present study, we took advantage of the temperature-sensitive growth defect of $\Delta \mathrm{pim} 1$ cells expressing $\mathrm{E}$. coli Lon ${ }^{\mathrm{K} 362 \mathrm{~A}}$ protease and isolated a multicopy suppressor that preserves mtDNA integrity in a pim1 null mutant. The respiratory competence of these cells remains impaired demonstrating a di rect involvement of PIM 1 protease in the biogenesis of the respiratory chain. Further analysis reveal ed deficiencies in the synthesis of mitochondrially encoded cytochrome b (Cob) and subunit I of the cytochrome c oxi dase (CoxI). PIM 1 function is required for the translation of mature COXI mRNA and the stability of $\mathrm{COXI}$ and $\mathrm{COB}$ transcripts containing multiple introns. Furthermore, pim1 mutants harboring mtDNA show deficiencies in the splicing of COXI and COB pre-mRNAs. Thus, the expression of mitochondrially encoded $\mathrm{COXI}$ and $\mathrm{COB}$ genes and thereby the assembly of respiratory chain complexes is under the proteolytic control of the ATP-dependent PIM 1 protease.

\section{Results}

A pim1 mutant harboring intact mtDNA is respiratory deficient

Defects in the integrity of mtDNA result in respiratory deficiency in yeast, as essential components of respira- tory chain complexes are mitochondrially encoded. The requirement of PIM 1 protease for the maintenance of mtDNA prevents, therefore, a characterization of its role in mitochondrial biogenesis. Genetic approaches, such as a search for multicopy suppressors of the pim1 null mutant phenotype, are hardly applicable because of the lack of mtDN A in these cells. To circumvent this problem, a $\Delta$ piml strain was employed which expresses E. coli Lon ${ }^{\mathrm{K} 362 \mathrm{~A}}$ protease in mitochondria ( $\Delta$ pim1/LON; Teichmann et al. 1996). The expression of Lon protease confers respiratory competence to the cells at $30^{\circ} \mathrm{C}$ but not at $36^{\circ} \mathrm{C}$ (Fig. 1; Teichmann et al. 1996). To investigate the function of PIM 1 protease, we performed a genetic screen for multicopy suppressors rescuing the conditional growth phenotype of $\Delta$ piml/LON cells. This search led to the identification of an extragenic suppressor (YEp13-SUP) that restored the growth of $\Delta \mathrm{pim1/}$ LON cells on nonfermentable carbon sources at $36^{\circ} \mathrm{C}$, that is, the integrity and expression of mtDNA ( $\Delta \mathrm{pim} 1 /$ LON / SUP) (Fig. 1).

To examine whether the suppressor al one stabilizes mtDNA in the absence of PIM 1 protease, the PIM 1 gene was disrupted in a haploid wild-type strain previously transformed with the rescuing plasmid YEp13-SUP ( $\Delta$ pim1/SUP). In contrast to pim1 null mutants, $\Delta$ piml/ SUP cells maintained mtDN A as demonstrated by crossing of these cells with a wild-type strain totally devoid of mtDNA. The resulting diploids were able to grow on nonfermentable carbon sources demonstrating the presence of intact mtDNA in $\Delta$ piml/SUP cells (data not shown). Although maintaining mitochondrial genome integrity, the suppressor did, however, not provide respi ratory competence to $\Delta$ piml cel ls lacking Lon protease (Fig. 1). Thus, independent of its role in stabilizing the

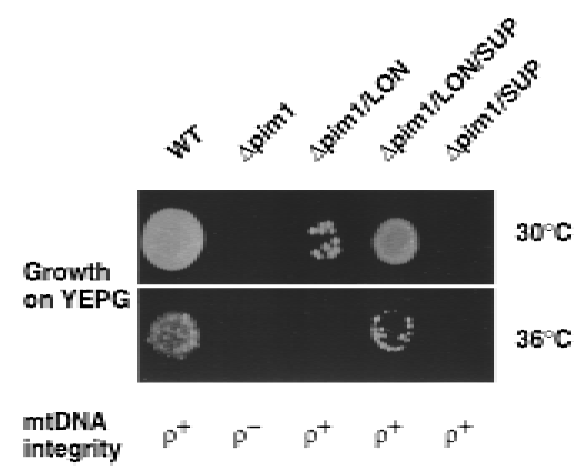

Figure 1. Respiratory deficiency of $\Delta$ pim 1 cells carrying intact mtDN A. Wild-type cells (WT), $\Delta$ piml cells ( $\Delta$ pim1), and $\Delta$ pim 1 cells complemented with the E. coli Lon protease ( $\Delta$ piml/ LON ), the suppressor gene ( $\Delta$ piml/SUP), or both ( $\Delta$ piml/LON / SUP), were grown in glucose-containing selective medium. Cells were harvested in exponential phase, spotted onto YEPG (rich medium containing $3 \%$ glycerol), and incubated at $30^{\circ} \mathrm{C}$ and $36^{\circ} \mathrm{C}$ for 7 and 10 days, respectively. MtDNA integrity $\left(\rho^{+}\right.$, $\left.\rho^{-}\right)$of various strains was examined by testing the respiratory competence of a diploid strain generated by mating with a $\rho^{0}$ PIM $1^{+}$strain. $\left(\rho^{+}\right)$Wild-type mtDNA; $\left(\rho^{-}\right)$mutant mtDNA carrying del etions. 
mitochondrial genome, PIM 1 function is required for the maintenance of the respiratory competence of the cells.

The rescuing plasmid, YEp13-SU P, contained a 5.4-kb insert from the right arm of chromosome IV bearing the genes SLU 7 (Frank and Guthrie 1992), YDR087c, encoding a protein of unknown function, and SSS1 (Esnault et al. 1993). Overexpression of Sss1p al one was sufficient to maintain mtDNA in the absence of PIM 1 protease. Disruption of the PIM1 gene in haploid cells expressing Sss1p from a multicopy plasmid did not impair the integrity of mtDN A (data not shown). Ssslp has originally been identified as a multicopy suppressor of the temperature-sensitive sec61-2 mutant (Esnault et al. 1993). It represents a subunit of Sec61p-complexes mediating the translocation of secretory proteins across the membrane of the endoplasmic reticulum (ER) (Esnault et al. 1994; Panzner et al. 1995; Finke et al. 1996). Therefore, an indirect effect on mtDNA metabolism seems likely. It should be noted, however, that a link between mitochondrial function and the ER was also suggested by studies on the yeast signal recognition particle (SRP) (Stirling and Hewitt 1992). The del etion of SRP subunits in yeast results in slow growing cells that are respiratory deficient, an observation whose functional significance remains to be demonstrated. In any case, the stabilization of mtDNA in pim1-null mutants overexpressing Ssslp enabled us to define novel functions of PIM 1 protease in mitochondria.

Defective synthesis of mitochondrially encoded Coxl and $\mathrm{Cob}$ in pim1 mutants

Seven subunits of respiratory complexes and one mitochondrial ribosomal subunit are encoded by mtDN A in yeast (Tzagol off and M yers 1986; Grivell and Schweyen 1989; Costanzo and Fox 1990). To investigate the essential role of PIM 1 for respiration, mitochondrially encoded proteins were labelled with $\left[{ }^{35}\right.$ S]methionine in $\Delta$ piml cells that maintain mtDNA because of the expression of the E. coli Lon protease ( $\Delta$ piml/LON ), the suppressor ( $\Delta \mathrm{piml} / \mathrm{SUP})$, or both ( $\Delta \mathrm{piml} / \mathrm{LON} / \mathrm{SUP})$ (Fig. 2A). Labeling of mitochondrially encoded proteins occurred with similar efficiencies in wild-type and $\Delta$ piml/LON cells, but incorporation of $\left[{ }^{35}\right.$ S]methionine was less efficient in $\Delta$ piml/SUP cells. The suppressor did not affect mitochondrial translation as indicated by the identical patterns of proteins synthesized in mitochondria of $\Delta$ piml/LON and $\Delta$ piml/LON/SUP cells (Fig. 2A).

N ewly synthesized ATP synthase subunits 6, 8, and 9 (Atp6, Atp8, and Atp9) and the ribosomal subunit Var1 accumulated at si milar levels in wild-type and in $\Delta$ piml cells containing mtDN A (Fig. 2A). In contrast, labeling of Coxl protein was strongly impaired in these cells ( $\Delta$ pim1/LON ; $\Delta$ pim1/SUP; Fig. 2A). Coxl did not accumulate at high levels in $\Delta$ piml/LON cells even when labeling was performed for longer time periods (Fig. 2B). Coxll and CoxlII, however, were synthesized in $\Delta$ pim1/ LON mitochondria, but degraded upon further incubation of the cells in pulse chase experiments (Fig. 2B).
Defects in cytochrome c oxidase assembly in the presence of limited concentrations of Coxl presumably result in the proteolysis of nonassembled CoxII and CoxIII (McEwen et al. 1986). N otably, the analysis of cell extracts by Western bl otting reveal ed the presence of CoxII in low amounts in $\Delta$ piml/LON but not in $\Delta$ piml/SUP cells (Fig. 2C). This finding is consistent with the pattern of growth on nonfermentable carbon sources at $30^{\circ} \mathrm{C}$ (see Fig. 1) and suggests the presence of low but functionally significant levels of Coxl in $\Delta$ piml/LON cells.

Interestingly, synthesis of Cob occurred at wild-type levels in $\Delta$ pim1/LON cells, whereas it was defective in mitochondria lacking a Lon-like protease ( $\Delta$ piml/SUP; Fig. 2A). Consistently, Cob protein was not detectable in $\Delta$ pim1/SUP cells upon Western blotting but accumulated, although at reduced levels, in $\Delta$ piml/LON cells (Fig. 2C). The presence of a Lon-like protease with reduced enzymatic activity in $\Delta$ piml mitochondria is apparently sufficient to maintain the expression of mitochondrially encoded $\mathrm{Cob}$, but not the efficient synthesis of Coxl. The impaired assembly of the Cox complex in $\Delta \mathrm{pim}$ //LON cells may indirectly cause slow degradation of newly synthesized Cob, thereby explaining the reduced amount of Cob in these cells. Similar observations have previously been reported for other respi ratory chain subunits (Rep and Grivell 1996). Taken together, these results point to a requirement of PIM 1 protease for the synthesis of Coxl and Cob and thereby explain the respiratory deficiency of $\Delta$ piml cells containing mtDNA.

To establish the dependence of Coxl synthesis on the proteolytic activity of PIM1, a proteolytically inactive mutant form of the protease was employed (PIM $1^{\mathrm{S1015A}}$ ): Replacement of the conserved serine 1015 by alanine abolishes the proteolytic activity of PIM 1 but does not affect the overall protein stability nor the ATP-dependent assembly of the homo-oligomeric protease (Rep et al. 1996b; Wagner et al. 1997). Wild-type and mutant protease were expressed in $\Delta$ piml/LON cells and mitochondrial protein synthesis was analyzed (Fig. 2D). Labeling of Coxl occurred in $\Delta$ piml/LON cells harboring active PIM 1 protease, but Coxl was hardly detectable in $\Delta \mathrm{pim} 1 /$ LON mitochondria in the presence of proteolytically inactive PIM 1. Thus, PIM 1-mediated proteolysis is required for the synthesis of CoxI in mitochondria.

PIM1 protease is required for intron-containing pre-mRNA stability and for translation of COXI mRNA

The defective synthesis of Coxl and Cob in pim1 mutants could result from impaired transcription or translation, or might reflect deficiencies in the stability or processing of the corresponding transcripts. As both genes harbor introns (Costanzo and Fox 1990; Pel and Grivell 1993, 1995), pre-mRNA splicing defects must also be considered.

We investigated the possibility of the PIM 1 function being related to the presence of introns in the $\mathrm{COXI}$ and COB gene. $\Delta$ piml/LON and $\Delta$ piml/SUP cells were converted to $\rho^{0}$ mutants and strains devoid of mitochondrial 


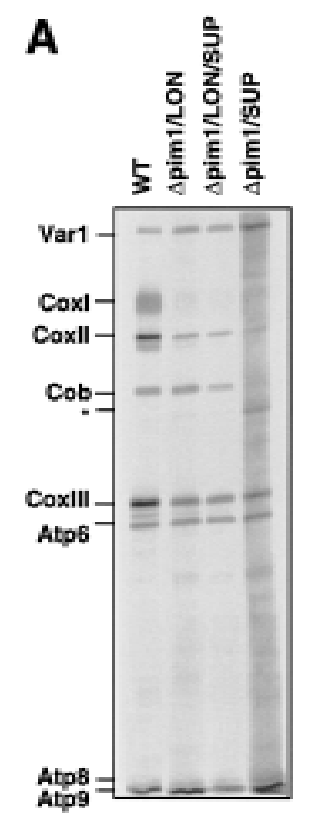

B
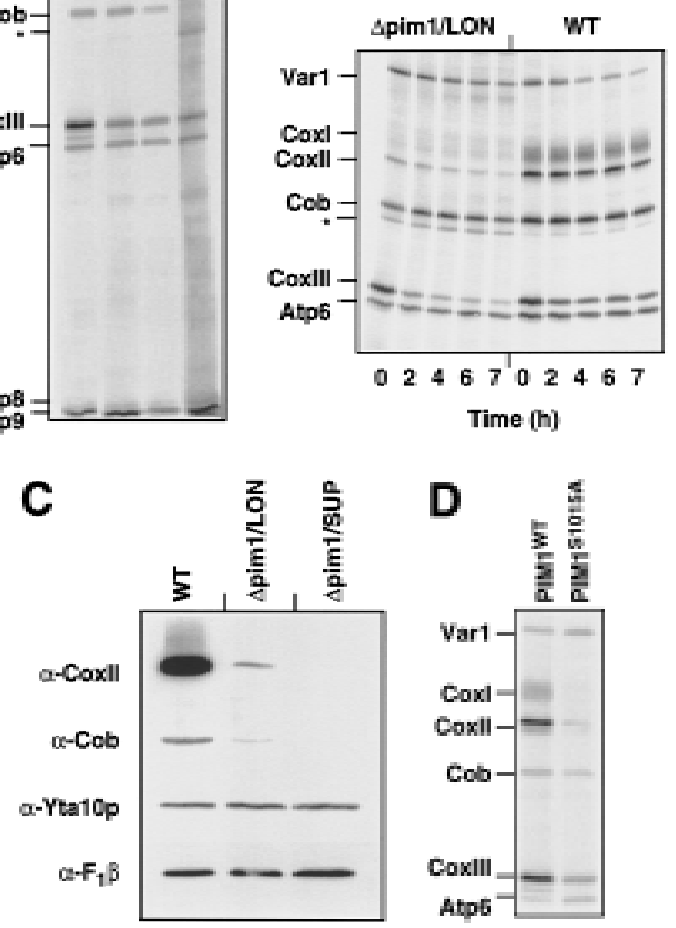

Figure 2. Requirement of PIMI protease for the synthesis of Coxl and Cob. (A) Synthesis of mitochondrially encoded proteins in vivo. Mitochondrial translation products were labeled with $\left[{ }^{35} \mathrm{~S}\right] \mathrm{methionine}$ in the presence of cycloheximide for 10 $\min (\mathrm{WT}, \Delta$ piml/LON , $\Delta$ piml/LON/SUP) or $30 \mathrm{~min}(\Delta \mathrm{pim} 1 /$ SUP) at $30^{\circ} \mathrm{C}$ in vivo as described in M aterials and M ethods and analyzed by SDS-PAGE. The translation efficiency was reduced in $\Delta$ piml/SUP cells. A band marked with an asterisk (*) is not strain-specific and was also detected in $\rho^{0}$ strains. (Coxl, Coxll, CoxIII) Subunits I, II, and III of cytochrome c oxidase, respectively; (Cob) cytochrome b; (Atp6, Atp8, Atp9) subunits 6, 8, and 9 of the $F_{0} F_{1}-A T P a s e$, respectively. (B) Degradation of newly synthesized Coxll and CoxIII in $\Delta$ piml/LON cells. Mitochondrially encoded polypeptides were synthesized in vivo for $30 \mathrm{~min}$ in the presence of $\left[{ }^{35}\right.$ S]methionine. After addition of cold methionine (10 mM ), cells were further incubated for the indicated time periods and then analyzed by SDS-PAGE. (C) Steady-state levels of Coxll and Cob in mitochondria lacking PIM 1 protease. Mitochondria were isolated from wild-type, $\Delta \mathrm{piml} / \mathrm{LON}$, and $\Delta$ pim1/SUP cells, subjected to SDS-PAGE and analyzed by Western blotting with polyclonal antisera directed against CoxII ( $\alpha$-CoxII), Cob $(\alpha-$ Cob), and Yta10p ( $\alpha$-Yta10p) and the $\beta$-subunit of the $F_{1}-A T P a s e$ $(\alpha-F 1 \beta)$ as gel loading controls. (D) Dependence of Coxl synthesis on PIM 1-mediated proteolysis. Labeling of mitochondrial translation products was performed for $10 \mathrm{~min}$ at $30^{\circ} \mathrm{C}$ in $\Delta$ piml/LON cells expressing wild-type PIM 1 or PIM $1^{\text {S1015A }}$ from multicopy plasmids. Wild-type and mutant protease accumulated at similar levels in mitochondria. introns were derived by cytoduction (Conde and Fink 1976; Berlin et al. 1991). This procedure allows the introduction of new mitochondrial information in a parent (cytoductant) that has conserved its nuclear genotype. Synthesis of Coxl occurred with similar efficiencies in wild-type and $\triangle \mathrm{pim1/LON}$ cells carrying intronless mtDNA (Fig. 3). Thus, in the presence of the E. coli Lon protease, removal of introns is sufficient to allow synthesis of $\mathrm{Cob}$ and Coxl. However, efficient synthesis of Cob but not Coxl was observed in cells carrying SUP but not LON (Fig. 3). These cells were respiratory deficient and di d not grow on nonfermentabl e carbon sources (data not shown). Thus, efficient expression of a COXI gene lacking introns still depends on PIM 1 or LON, suggesting defects in Coxl translation or mRNA stability in cells devoid of a Lon-like protease.

To distinguish between these possibilities, mitochondrial RNA (mtRNA) was isolated from wild-type and from $\Delta$ pim1/SUP cells carrying intronless $m$ tDNA and analyzed by Northern blot hybridization with probes specific for COXI and COB exons (Fig. 4A). With wildtype cells, the probes hybridized with transcripts of $\sim 2.1$ and $2.2 \mathrm{~kb}$, which correspond to mature $\mathrm{COXI}$ and $\mathrm{COB}$ mRNA, respectively (Fig. 4A). Similarly, mature-sized COXI and COB transcripts were detected in $\triangle \mathrm{piml}$ /SUP cells harboring intronless mtDNA (Fig. 4A). COXI mRN A accumulated in significantly increased amounts in $\Delta$ pim1/SUP cells devoid of mitochondrial introns when compared to wild-type cells (Fig. 4A). N evertheless, Coxl protein was not synthesized in these cells (see Fig. 3) demonstrating the requirement of PIM 1 protease for efficient translation of mature COXI transcripts.

COXI and COB transcripts were not detected in $\Delta$ piml/SUP cells with an intron-containing mitochondrial genome (Fig. 4A), although transcription proceeded normally in these cells (see below). A pparently, PIM 1

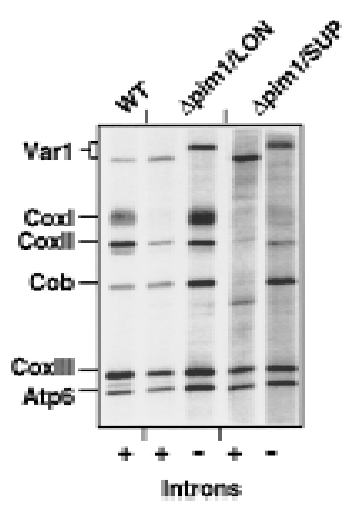

Figure 3. Mitochondrial protein synthesis in $\Delta$ piml cells carrying intronless mtDNA. $\Delta$ piml/LON and $\Delta$ pim1/SUP cells devoid of mitochondrial introns were generated by cytoduction. Mitochondrial translation products were labeled with [35 S]methionine for $10 \mathrm{~min}(\mathrm{WT} ; \Delta \mathrm{pim} 1 / \mathrm{LON}$ ) or $30 \mathrm{~min}$ ( $\Delta \mathrm{pim} 1 / \mathrm{SUP})$ at $30^{\circ} \mathrm{C}$ in vivo and analyzed by SDS-PAGE. The difference in the electrophoretic mobility of Varl reflects gene polymorphism in the different mitochondrial genomes (Butow et al. 1985). 
PIM1 protease and mitochondrial gene expression

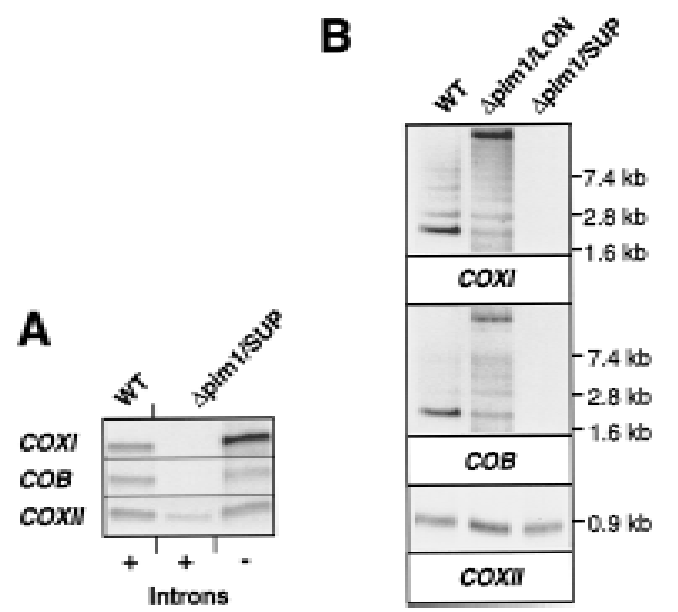

Figure 4. Northern blot analysis of $\mathrm{COXI}$ and $\mathrm{COB}$ transcripts in mitochondria lacking PIM 1 protease. (A) mtRN A was isolated from wild-type (WT) and $\Delta$ piml/SUP cells, harboring an intron-containing or an intronless mitochondrial genome (tintrons), and analyzed with COXI and COB-specific exon probes and a COXII probe for control as described in Materials and Methods. (B) mtRN A from wild-type (WT), $\Delta$ pim1/LON, and $\Delta$ piml/SUP mitochondria with intron-containing mtDN A was analyzed as in A.

protease is required for the stability of $\mathrm{COXI}$ and $\mathrm{COB}$ transcripts harboring multiple introns. It is, however, conceivable that processing defects in the absence of PIM 1 cause the degradation of $\mathrm{COB}$ and COXI transcripts, as previous studies reveal ed a correlation between mRNA processing defects and transcript degradation (Grivell 1995).

PIM 1 protease affects $\mathrm{COXI}$ and $\mathrm{COB}$ premRNA processing

To further analyze a possible role of PIM 1 protease in pre-mRNA processing, we again took advantage of the observation that E. coli Lon protease with reduced enzymatic activity is sufficient to maintain the respiratory competence of pim1 null mutant cells at $30^{\circ} \mathrm{C}$. RN A was isolated from wild-type and $\Delta$ piml/LON mitochondria and subjected to $\mathrm{N}$ orthern blot analysis with probes specific for exons of $\mathrm{COXI}$ or $\mathrm{COB}$ (Fig. 4B). In contrast to $\Delta$ piml/SUP cells, COXI and COB transcripts accumulated in $\Delta \mathrm{pim} 1 / \mathrm{LON}$ cells. When compared to wild-type cells, however, a significant decrease in the amounts of mature transcripts and an increase of larger precursor transcripts were detected with $\mathrm{COXI}$ - and COB-specific probes in $\Delta$ pim1/LON cells (Fig. 4B). Apparently, the presence of a Lon-like protease with reduced enzymatic activity in mitochondria is sufficient to stabilize COXI and $\mathrm{COB}$ transcripts containing multiple introns, but does not allow efficient RN A processing to occur. These results suggest an involvement of PIM 1 protease in splicing processes in mitochondria. $\mathrm{N}$ otably, despite the presence of reduced levels of mature COB transcripts, Cob synthesis was hardly affected in $\Delta$ pim1/LON cells (see Fig. 2A).

mtRNA of wild-type and $\Delta$ piml/SUP cells was analyzed with probes specific for introns of group II. In contrast to group I introns, these introns form stable lariat structures upon splicing and can therefore be detected by Northern blot hybridization (Costanzo and Fox 1990; Perlman 1990). Probes specific for the first intron of COB (bl1) or the last intron of COXI (al5y) hybridized to transcripts of $\sim 0.8 \mathrm{~kb}$ from wild-type and $\Delta$ pim1/SUP mitochondria; these species correspond in size to the excised lariat forms (Fig. 5). Interestingly, excised intron bl 1 accumulated at higher levels in $\Delta$ piml/SUP cells, most likely indicating an upregulation of transcription because of the impaired synthesis of $\mathrm{Cob}$ in these cells. These findings confirm transcription of $\mathrm{COB}$ and $\mathrm{COXI}$ in $\triangle \mathrm{pim} 1 /$ SUP cells and demonstrate that PIM 1 protease is not required for the splicing of these group II introns.

$M$ any mitochondrial introns contain an open reading frame that encodes an mRNA maturase fused in frameto the preceding exon (Costanzo and Fox 1990; Pel and Grivell 1993; Grivell 1995). Splicing of these introns is catalyzed by the intron-encoded maturase and thus depends on its synthesis. Analyzing mtRN A from $\Delta \mathrm{pim} /$ SUP cells, no excised lariat structure was detectable with a probe specific for intron 1 of COXI (al1), a 2.4-kb group II intron that encodes an mRN A maturase (Fig. 5). The deficiency in the splicing of this maturase-encoding intron is in agreement with the observed requirement of PIM 1 protease for Coxl translation. $\mathrm{N}$ otably, a defect in the splicing of intron al 1 did not impai $r$ the processing of the downstream intron al5y, which does not encode a maturase (Fig. 5). Indeed, cotranscriptional splicing has been demonstrated for introns that do not encode a maturase (Lewin et al. 1995). The excised intron al5y, however, accumulated at a reduced level in $\Delta$ pim1/SUP mitochondria when compared to wild type, most likely caused by rapid degradation of nonprocessed COXI premRNA.

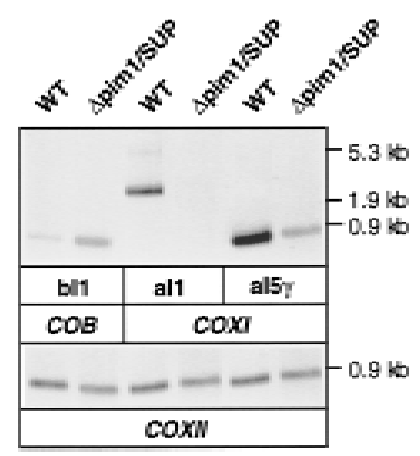

Figure 5. Characterization of $\mathrm{COXI}$ and $\mathrm{COB}$ pre-mRNA processing defects in pim 1 mutants by $\mathrm{N}$ orthern hybridization using intron-specific probes. mtRN A isolated from wild-type (WT) and $\Delta$ piml/SUP cells was analyzed with intron-specific COB and COXI probes. DNA probes specific for the group II introns bl 1 of COB, al 1 and al $5 \gamma$ of $C O X I$ and COXII, as a control, were employed. 


\section{Discussion}

Cells lacking PIM 1 protease lose the integrity of mtDN A and thereby their respiratory competence (Suzuki et al. 1994; van Dyck et al. 1994). This phenotype has prevented a detailed characterization of the role of PIM 1 protease in mitochondrial biogenesis. In the present manuscript, we took advantage of the identification of a multicopy suppressor that stabilizes mtDNA in the absence of PIM 1. The analysis of pim 1 mutants containing mtDNA revealed deficiencies in the synthesis of mitochondrially encoded Coxl and Cob and thereby in the assembly of respiratory chain complexes. The defect in the synthesis of Coxl and Cob provides an explanation for the observed respiratory deficiency of pim1 mutant cells containing intact mtDNA. These results establish essential proteolytic functions of the ATP-dependent PIM 1 protease in mitochondria that control the biogenesis of the respi ratory chain (summarized in Fig. 6).

PIM 1 protease is required for the translation of mature COXI mRN A. Synthesis of Coxl was impaired in $\Delta$ pim 1 cells carrying intronless mtDNA, although mature mRN A accumulated in these cells. Coxl synthesis was

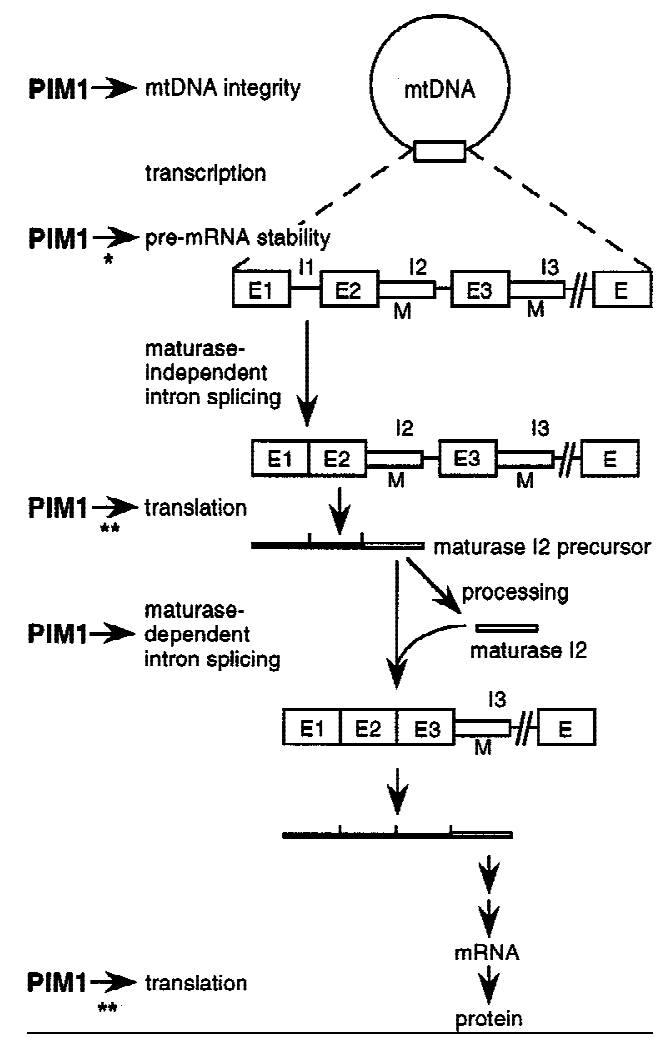

Figure 6. Roles of PIM 1 protease in mitochondrial biogenesis. PIM 1-mediated proteolysis is required for mtDN A integrity and the expression of the intron-containing COXI and COB genes in mitochondria (see text for details). (*) The instability of preRNA in the absence of PIM 1 may be a secondary effect of premRNA processing deficiencies. (**) PIM 1 protease is only required for translation of COXI mRNA. (E, E1-3) exons; (I, I1-3) introns; (M) mRNA maturases. not restored upon expression of a proteolytical ly inactive PIM 1 mutant, demonstrating the requirement of PIM 1mediated proteolysis for Coxl translation. Membranebound translational activator proteins have been identified in mitochondria (Fox 1996; Rödel 1997). They physically interact with mitochondrial ribosomal subunits and the 5' untransl ated leader of their target and thereby allow the post-transcriptional control of gene expression (Haffter et al. 1991; Mulero and Fox 1993; Brown et al. 1994). Activator proteins are mRNA-specific and regulate the synthesis of mitochondrially encoded proteins in a gene-specific manner (Pel and Grivell 1994; Fox 1996). Similarly, PIM 1 protease is required for the transl ation of COXI but not of other mitochondrially encoded proteins. Furthermore, PIM 1 was found in association with the inner surface of the mitochondrial inner membrane after sonication of isol ated mitochondria (L.van Dyck, I. Wagner, and T. Langer, unpubl.). It is conceivable that PIM 1 exerts its function in Coxl synthesis by regulating the activity of other mitochondrial proteins. This might include the activation of proteins specifically involved in the translation of the COXI gene by PIM 1-mediated processing. Alternatively, PIM 1 protease might be required to degrade specific RNA-binding proteins that inhibit the translation of COXI transcripts.

Several introns of the $C O X I$ and $C O B$ genes contain an open reading frame that encodes an mRNA maturase fused in frame to the preceding exon (Costanzo and Fox 1990; Pel and Grivell 1993, 1995). Splicing of these introns is catalyzed by the intron-encoded maturase and thus depends on its translation. The deficiency in COXI pre-mRNA splicing in piml mutants can therefore be attributed to the impaired synthesis of intron-encoded mRN A maturases. Moreover, the failure to remove introns in the absence of PIM 1 protease may result in the rapid degradation of COXI pre-mRNA transcripts, as a linkage between RNA processing and stability has been observed in mitochondria of various organisms (Grivell 1995). Thus, deficiencies in COXI pre-mRNA stability and splicing can be explained satisfactorily by the requirement of PIM1 protease for Coxl translation. The pleiotropic effect of pim1 mutants on the expression of the COXI gene is reminiscent of other proteins involved in mitochondrial gene expression (Groudinsky et al. 1993; Manthey and McEwen 1995). The product of the yeast nuclear gene PET309 is required for the translation of mature COXI and the stability of COXI pre-mRNA, as is PIM 1 protease (Manthey and McEwen 1995). In contrast to pim1 cells, however, COB transcripts are not affected in pet309 mutants. Furthermore, SU V3, encoding a putative RN A helicase, is necessary for the stability of intron-containing $\mathrm{COXI}$ and $\mathrm{COB}$ transcripts, but not for the translation of mature COXI (Golik et al. 1995).

The analysis of COB gene expression in pim1 mutant cells, however, points to additional functions of PIM 1 protease in mitochondrial gene expression. In contrast to COXI, translation of mature COB mRNA does not depend on the presence of PIM 1 in mitochondria as demonstrated by the efficient synthesis of Cob in $\Delta$ piml/ SUP cells carrying an intronless mitochondrial genome 
and in $\Delta$ pim1/LON cells. Indirect effects on the splicing of $\mathrm{COB}$ transcripts because of impaired synthesis of intron-encoded mRNA maturases can therefore be excluded. Still, $N$ orthern bl ot analysis of $\Delta$ pim1/LON cells harboring a Lon-like protease with reduced enzymatic activity revealed deficiencies in the processing of $\mathrm{COB}$ transcripts, indicating a role of PIM 1 protease for the splicing of COB pre-mRN As. N otably, the maturase encoded by the intron bl 4 of the $\mathrm{COB}$ gene is required for the splicing of both intron bl 4 itself and intron al 4 of the COXI gene (Dhawale et al. 1981; Banroques et al. 1987). Defects in the processing of $\mathrm{COB}$ pre-mRNAs result therefore in an impaired splicing of COXI transcripts.

How may PIM 1 affect the splicing of mitochondrial transcripts? PIM 1 protease could regulate the activity or stability of a protein directly involved in the splicing process. It is, for instance, an attractive possibility that PIM 1 mediates the proteolytic processing of some mRN A maturases that are synthesized as fusion proteins with the peptide products of preceding exons (Costanzo and Fox 1990; Pel and Grivell 1993; Grivell 1995). Indeed, an energy-dependent step in the splicing of intron bl 4 of the COB gene has been proposed (M uroff and Tzagol off 1990). Further studies, however, are necessary to substantiate this hypothesis.

The stability of COXI and COB mRNAs containing multiple introns was impaired in $\Delta \mathrm{piml}$ cells lacking a Lon-like protease but containing mtDNA. Impaired splicing may result in the degradation of the intron-containing transcripts. Otherwise a direct role of PIM 1 protease for premRNA stability has to be envisioned. It should be noted in this context that expression of Lon protease did result in the stabilization of $\mathrm{COXI}$ and $\mathrm{COB}$ premRNAs in $\Delta \mathrm{piml}$ cells, although splicing was impaired in these cells.

Although our results assign crucial functions to PIM 1 for COXI and COB pre-mRN A stability and COXI translation, the protease is not required for transcription of these genes. The Northern blot analysis of $\Delta \mathrm{piml}$ cells harboring intact mtDN $A$ with intron-specific probes revealed normal transcription of $\mathrm{COXI}$ and $\mathrm{COB}$ genes in these cells. Thus, the lack of transcripts in the absence of PIM 1 is caused by degradation of COXI and COB premRN As. As most mitochondrially encoded genes, COXI and $\mathrm{COB}$ are initially transcribed into polycistronic RNAs followed by the processing of the primary transcript (Grivell 1989). The COXI gene is cotranscribed with the genes encoding ATP synthase subunits 6 and 8 , synthesis of which occurred at wild-type levels in mitochondria lacking PIM 1 protease. Similarly, the COB gene is transcribed into a precursor also containing tRNA Glu, which is essential for the synthesis of all mitochondrially encoded proteins. Thus, polycistronic precursor processing does not depend on the presence of PIM 1 protease in mitochondria.

PIM 1 protease has recently been proposed to serve as a chaperone in the assembly of respiratory complexes independent of its proteolytic activity (Rep et al. 1996b). Our results do not exclude chaperone-like properties of PIM 1, however, they explain the respiratory deficiency of piml mutant cells by the lack of essential proteolytic functions of PIM 1. PIM 1-mediated proteolysis is required for mtDN A integrity (Wagner et al. 1997) and for the synthesis of respiratory chain subunits. Thus, impaired respiration in the absence of PIM 1 protease is not caused by deficiencies in the assembly process per se or misfol ded polypeptides accumulating within mitochondria, but reflects specific requi rements of PIM 1-mediated proteolysis for the biogenesis of the respiratory chain. The homologous Lon protease from E. coli can partially substitute for PIM 1 in these processes, suggesting a conserved mode of action.

\section{Materials and methods}

Yeast strains and genetic analysis

Yeast strains used in this study are described in Table 1. The wild-type strain YPH500 contains long gene variants of COXI and COB. Cells were grown on YEP medium (1\% yeast extract, $2 \%$ peptone) or on minimal medium $(0.7 \%$ yeast nitrogen base containing ammonium sulfate) that was supplemented with the auxotrophic requirements and contained glucose $(2 \%)$, galactose $(2 \%)$, or glycerol $(3 \%)$ as the sole carbon source.

The genetic analysis of yeast mutants was carried out according to published procedures (Sherman 1991). $\rho^{0}$ derivative strains were prepared by ethi dium bromide treatment (Fox et al. 1991). Cytoduction was performed essentially as described (Conde and Fink 1976; Berlin et al. 1991): $\rho^{0}$ derivatives were transformed with plasmids expressing the suppressor gene and crossed with karl strains bearing the mitochondrial genome of interest. Cytoductants were selected both for their ability to grow on YEP glycerol and the presence of auxotrophic markers of the $\rho^{0}$ parental strain. Mutant cytoductants were generated by disruption of the PIM1 gene using a pim1::HIS3 disruption cassette (Wagner et al. 1997).

Table 1. Yeast strains used in this study

\begin{tabular}{|c|c|c|}
\hline Strain & Genotype & Source \\
\hline YPH500 & MAT $\alpha$ ura3-52 lys2-801 ${ }^{\text {amber }}$ ade101 ${ }^{\text {ochre }} \operatorname{trp} 1-\Delta 63$ his3-200 leu2- $\Delta 1\left(\rho^{+}\right)$ & Sikorski and Hieter (1989) \\
\hline$\Delta \mathrm{pim} 1 / \mathrm{LON} *$ & MAT $\alpha$ pim::HIS3 $\left(\rho^{+}\right)$pVT 100-U ::ADH1-Su9(69)Lon ${ }^{\text {K362A }: 2 \mu, ~ U R A 3 ~}$ & Teichmann et al. (1996) \\
\hline$\Delta \mathrm{piml} / \mathrm{LON} / \mathrm{SUP} *$ & $\begin{array}{l}\text { MAT } \alpha \text { pim1::HIS3 }\left(\rho^{+}\right) \text {pVT 100-U::ADH 1-Su9(69)Lon }{ }^{\text {K362A }: ~} 2 \mu \text {, URA3, } \\
\text { YEp13-SUP: } 2 \mu \text {, LEU } 2\end{array}$ & this study \\
\hline$\Delta$ piml/SUP* & MAT $\alpha$ pim1::HIS3 $\left(\rho^{+}\right)$YEp13-SUP: $2 \mu$ LEU 2 & this study \\
\hline kar167-1 & MATa kar1-1 trp5 ( $\rho^{+}$intronless) & Seraphin et al. (1987) \\
\hline
\end{tabular}

Strains labeled with an asterisk are isogenic to $\mathrm{YPH} 500$. 


\section{Nucleic acid procedures}

Standard DNA manipulations were carried out as previously described (Sambrook et al. 1989; Ausubel et al. 1992). Doublestranded DN A templates were sequenced using Sequenase (USB Corp.) according to the manufacturer's guidelines. mtRN A was extracted essentially as described (Schmitt et al. 1990). Mitochondria were isolated according to published procedures (Herrmann et al. 1994; Zinser and Daum 1995) and lysed at a concentration of $10 \mathrm{mg} / \mathrm{ml}$ in $50 \mathrm{~mm}$ Tris- $\mathrm{HCl}(\mathrm{pH} 7.4), 10 \mathrm{~mm}$ EDTA, $1 \%(\mathrm{wt} / \mathrm{vol})$ SDS in the presence of proteinase $\mathrm{K}(100$ $\mu \mathrm{g} / \mathrm{ml}$ ). After addition of $\mathrm{NaCl}$ to a concentration of $150 \mathrm{~mm}$, mtRN A was phenol-extracted. N orthern blotting of mtRN A (3 $\mu \mathrm{g}$ ) was performed with Hybond-N nylon membrane (Amersham Corp.) using the protocol of the vendor. Probes were label ed with $\left[\alpha{ }^{32} \mathrm{P}\right] \mathrm{dATP}$ using the Random Prime DN A labeling kit (Boehringer $M$ annheim). Hybridization was carried out for $15 \mathrm{hr}$ at $42^{\circ} \mathrm{C}$ in $5 \times \mathrm{SSC}, 0.5 \%$ (wt $/ \mathrm{vol}$ ) SDS, $40 \%$ formamide, $5 \times$ Denhardt's reagent, and $20 \mathrm{mg} / \mathrm{ml}$ denatured salmon sperm DN A. M embranes were washed three times with $2 \times$ SSC, $0.5 \%$ (wt/ vol) SDS, at room temperature for $5 \mathrm{~min}$ and twice in $1 \times$ $\mathrm{SSC}, 0.5 \% \mathrm{SDS}$, at $50^{\circ} \mathrm{C}$ for $30 \mathrm{~min}$. The indicated sizes of the transcripts were estimated using the RNA molecular weight marker II (Boehringer M annheim).

The following DNA fragments were used as probes for the $\mathrm{N}$ orthern blot analysis: COB exon probe, pA 12/M b2 (N obrega and Tzagoloff 1980); COXI exon probe, pCOX1/A4-I corresponding to a DNA fragment from COXI containing exon A4 and part of intron al 4 (kindly provided by A. Tzagol off, Columbia University, New York, NY); COXII probe, PCR-amplified 689-bp internal DNA fragment of COXII. COB intron probes: bl1, pYJL12; bl2, pYJL5 (Lazowska et al. 1989); COXI intron probes: al1, 766 bp HinDII-Mbol fragment in pUC13; al5y, pYJL14, 533-bp Taql-Rsal fragment in pUC13 kindly provided by J. Lazowska (CNRS, Gif-Sur-Yvette, France).

\section{Isolation of the multicopy supressor gene}

A YEp13 yeast genomic library was used to transform $\Delta$ piml/ LON cells to leucine prototrophy (Broach et al. 1979). U ra3 ${ }^{+}$ Leu2 $^{+}$transformants were replica plated on YEP glycerol and incubated at $36^{\circ} \mathrm{C}$ for 7 days. YEp13-SUP was selected for its ability to rescue the thermosensitive growth defect of $\Delta \mathrm{piml} /$ LON cells. Plasmid linkage of the suppression was confirmed by retransformation. The insert extremities of the rescuing plasmid YEp13-SUP were sequenced using primers YEP13a (5'GCTTCGCTACTTGGAG-3') and YEP13b (5'-ATCGGTGATGTCGGCG-3'). A search for homology using the BLAST program led to the identification of a 5.4-kb DNA fragment on the right arm of chromosome IV encoding SSS1, YDR087c, and SLU 7. The suppressive effect of Ssslp was demonstrated by transforming the multicopy plasmid pTX64 harboring SSS1 (kindly provided by T. Sommer, M ax-Delbrück-Center, Berlin, Germany) in the wild-type strain YPH500 and subsequent disruption of the PIM 1 gene.

\section{Labeling of mitochondrial translation products in vivo}

Mitochondrial translation products were labeled in vivo essentially as described (Dougl as et al. 1979; M cKee and Poyton 1984; Langer et al. 1995). Cells were grown in minimal medium galactose lacking methionine. For each time point, cells (0.5 $\mathrm{OD}_{578}$ units) were harvested in midexponential phase by $15-\mathrm{sec}$ centrifugation in a bench centrifuge, washed and resuspended in $500 \mu \mathrm{l}$ of labeling buffer ( $40 \mathrm{~mm} \mathrm{~K}_{2} \mathrm{HPO}_{4}$ at $\mathrm{pH} 6,2 \%$ galactose). Cells were incubated for $10 \mathrm{~min}$ at $30^{\circ} \mathrm{C}$ and cycloheximide was added to a final concentration of $150 \mu \mathrm{g} / \mathrm{ml}$ to inhibit the cytosolic protein synthesis. After a further incubation for $2 \mathrm{~min}$, labeling of translation products with $\left.{ }^{35} \mathrm{~S}\right]$ methionine $(40 \mu \mathrm{Ci}$; $1000 \mathrm{Ci} / \mathrm{mmole}$ ) was performed for the times indicated and stopped by the addition of unlabeled methionine ( $10 \mathrm{~mm}$ ). Cells were isolated by 15 -sec centrifugation and washed with $10 \mathrm{~mm}$ methionine. Total cell proteins were extracted by al kaline lysis (Y affe and Schatz 1984) and solubilized by shaking for $30 \mathrm{~min}$ at $4^{\circ} \mathrm{C}$ in LiDS sample buffer (2\% lithium dodecylsulfate, $10 \%$ glycerol, 2.5\% $\beta$-mercaptoethanol, 0.02\% bromphenol blue, 60 $\mathrm{mm} \mathrm{Tris} / \mathrm{Cl}$ at $\mathrm{pH}$ 6.8). Proteins were separated by SDS-PAGE and visualized by autoradiography.

For pulse chase experiments, cells ( $3 O D_{578}$ units) were resuspended in labeling buffer $(1.5 \mathrm{ml})$. After addition of cycloheximide, labeling was performed for $30 \mathrm{~min}$ at $30^{\circ} \mathrm{C}$ with [ $\left.{ }^{35} \mathrm{~S}\right] \mathrm{me}-$ thionine $(100 \mu \mathrm{Ci}, 1000 \mathrm{Ci} / \mathrm{mmole})$. After addition of methionine $(10 \mathrm{~mm})$, reisolation and washing, cells were resuspended in labeling medium $(600 \mu \mathrm{l})$ contai ning methionine $(10 \mathrm{~mm})$ and further incubated at $30^{\circ} \mathrm{C}$. At the time points indicated, al iquots of the cells were harvested and analyzed.

\section{Acknowledgments}

We thank J. Lazowska, T. Sommer, A. Tzagol off, and C. Jacq for plasmids and yeast strains, and we are grateful to J. Lazowska for stimulating discussions. The excellent technical assistance of Gabi Ludwig, Petra Robisch, and Alexandra Weinzierl is gratefully acknowledged. L.v.D. was a recipient of a Senior $\mathrm{Re}$ search Fellowship of the European Union (DGXII; biotechnology). The work was supported by grants from the Deutsche Forschungsgemeinschaft (La918/1-2; SFB184, B21) to T.L.

The publication costs of this article were defrayed in part by payment of page charges. This article must therefore be hereby marked "advertisement" in accordance with 18 USC section 1734 solely to indicate this fact.

\section{References}

Adam, Z. 1996. Protein stability and degradation in chloroplasts. Plant Mol. Biol. 32: 773-783.

Arlt, H., R. Tauer, H. Feldmann, W. Neupert, and T. Langer. 1996. The YTA 10-12-complex, an AAA protease with chaperone-like activity in the inner membrane of mitochondria. Cell 85: 875-885.

Ausubel, F.J., R. Brent, R.E. Kingston, D.D. Moore, J.G. Seidman, J.A. Smith, and K. Struhl 1992. Current protocols in molecular biology. Greene Publishing Associates and Wiley-Interscience, N ew York, NY.

Banroques, J., J. Perea, and C. Jacq. 1987. Efficient splicing of two yeast mitochondrial introns controlled by a nuclear-encoded maturase. EMBO J. 6: 1085-1091.

Baumeister, W. and A. Lupas. 1997. The proteasome. Curr. Opin. Struct. Biol. 7: 273-282.

Berlin, V., J.A. Brill, J. Trueheart, J.D. Boeke, and G.R. Fink. 1991. Genetic screens and selections for cell and nuclear fusion mutants. Methods Enzymol. 194: 774-792.

Broach, J.R., J.N . Strathern, and J.B. Hicks. 1979. Transformation in yeast: Development of a hybrid cloning vector and isolation of the CAN1 gene. Gene 8: 121-133.

Brown, N.G., M.C. Costanzo, and T.D. Fox. 1994. Interactions among three proteins that specifically activate translation of the mitochondrial COX3 mRNA in Saccharomyces cerevisiae. Mol. Cell. Biol. 14: 1045-1053.

Butow, R.A., P.S. Perlman, and L.I. Grossman. 1985. The unusual var1 gene of yeast mitochondrial DNA. Science 228: 1496-1501.

Campbell, C.L., N. Tanaka, K.H. White, and P.E. Thorsness. 
1994. Mitochondrial morphological and functional defects in yeast caused by ymel are suppressed by mutation of a $26 \mathrm{~S}$ protease subunit homologue. Mol. Biol. Cell 5: 899-905.

Conde, J. and G.R. Fink. 1976. A mutant of Saccharomyces cerevisiae defective for nuclear fusion. Proc. Natl. Acad. Sci. 73: 3651-3655.

Confal onieri, F. and M. Duguet. 1995. A 200-amino acid ATPase module in search of a basic function. BioEssays 17: 639-650.

Costanzo, M.C. and T.D. Fox. 1990. Control of mitochondrial gene expression in Saccharomyces cerevisiae. Annu. Rev. Genet. 24: 91-113.

Coux, O., K. Tanaka, and A.L. Goldberg. 1996. Structure and functions of the $20 \mathrm{~S}$ and $26 \mathrm{~S}$ proteasomes. Annu. Rev. Biochem. 65: 801-847.

Dhawahle, S., D.K. Hanson, N.J. Alexander, P.S. Perlman, and H.R. Mahler. 1981. Regulatory interactions between mitochondrial genes: Interactions between two mosaic genes. Proc. Natl. Acad. Sci. 78: 1778-1782.

Douglas, M., D. Finkelstein, and R.A. Butow. 1979. A nalysis of products of mitochondrial protein synthesis in yeast: $\mathrm{Ge}$ netic and biochemical aspects. Methods Enzymol. 56: 58-66.

Esnault, Y., M.O. Blondel, R.J. Deshaies, R. Schekman, and F. Kepes. 1993. The yeast SSS1 gene is essential for secretory protein translocation and encodes a conserved protein of the endoplasmic reticulum. EMBO J. 12: 4083-4093.

Esnault, Y., D. Feldheim, M.O. Blondel, R. Schekman, and F. Képès. 1994. SSS1 encodes a stabilizing component of the Sec61 subcomplex of the yeast protein transl ocation apparatus. J. Biol. Chem. 269: 27478-27485.

Finke, K., K. Plath, S. Panzner, and S. Prehn. 1996. A second trimeric complex containing homologs of the Sec61p complex functions in protein transprot across the ER membrane of S. cerevisiae. EMBO J. 15: 1482-1494.

Fox, T.D. 1996. Translational control of endogenous and recoded nuclear genes in yeast mitochondria: Regulation and membrane targeting. Experientia 52: 1130-1135.

Fox, T.D., L.S. Folley, J.J. Mulero, T.W. McMullin, P.E. Thorsness, L.O. Hedin, and M.C. Costanzo. 1991. Analysis and manipulation of yeast mitochondrial genes. Methods Enzymol. 194: 149-165.

Frank, D. and C. Guthrie. 1992. An essential splicing factor, SLU 7, mediates 3' splice site choice in yeast. Genes \& Dev. 6: 2112-2124.

Goldberg, A.L. 1992. The mechanism and functions of ATPdependent proteases in bacterial and animal cells. Eur. J. Biochem. 203: 9-23.

Golik, P., T. Szczepanek, E. Bartnik, P.P. Stepien, and J. Lazowska. 1995. The S. cerevisiae nuclear gene SU V3 encoding a putative RN A helicase is necessary for the stability of mitochondrial transcripts containing multiple introns. Curr. Genet. 28: 217-224.

Gottesman, S. and M.R. M aurizi . 1992. Regulation by proteolysis: Energy-dependent proteases and their targets. Microbiol. Rev. 56: 592-621.

Grivell, L.A. 1989. Nucleo-mitochondrial interactions in yeast mitochondrial biogenesis. Eur. J. Biochem. 182: 477-493.

- - . 1995. Nucleo-mitochondrial interactions in mitochondrial gene expression. Crit. Rev. Biochem. Mol. Biol. 30: 121-164.

Grivell, L.A. and R.J. Schweyen. 1989. RNA splicing in yeast mitochondria: Taking out the twists. Trends Genet. 5: 3941.

Groudinsky, O., I. Bousquet, M.G. Wallis, P.P. Slonimski, and G. Dujardin. 1993. The NAM 1/MTF2 nuclear gene product is selectively required for the stability and/or processing of mitochondrial transcripts of the atp 6 and of the mosaic, cox 1 and cytb genes in Saccharomyces cerevisiae. Mol. \& Gen. Genet. 240: 419-427.

Guélin, E., M. Rep, and L.A. Grivell. 1994. Sequence of the AFG3 gene encoding a new member of the FtsH/Y mel/T ma subfamily of the AAA-protein family. Yeast 10: 1389-1394.

Guélin, E., M. Rep, and L.A. Grivell. 1996. Afg3p, a mitochondrial ATP-dependent metalloprotease, is involved in the degradation of mitochondrially-encoded Cox1, Cox3, Cob, Su6, Su8 and Su9 subunits of the inner membrane complexes III, IV and V. FEBS Lett. 381: 42-46.

Haffter, P., T.W. M cM ullin, and T.D. Fox. 1991. Functional interactions among two yeast mitochondrial ribosomal proteins and an mRNA-specific translational activator. Genetics 127: 319-326.

Herrmann, J.M., H. Fölsch, W. N eupert, and R.A. Stuart. 1994. Isolation of yeast mitochondria and study of mitochondrial protein translation. In Cell biology: A laboratory handbook (ed. D.E. Celis), pp. 538-544. Academic Press, San Diego, CA.

Hilt, W. and D. Wolf. 1996. Proteasomes: Destruction as a programme. Trends Biochem. Sci. 21: 96-102.

Kunau, W.H., A. Beyer, T. Franken, K. Gotte, M. Marzioch, J. Sai dowsky, A. Skal etz-Rorowski, and F.F. Wiebel. 1993. T wo complementary approaches to study peroxisome biogenesis in Saccharomyces cerevisiae: Forward and reversed genetics. Biochimie 75: 209-224.

Langer, T. and W. N eupert. 1996. Regulated protein degradation in mitochondria. Experientia 52: 1069-1076.

Langer, T., A. Pajic, I. Wagner, and W. Neupert. 1995. Proteolytic breakdown of membrane-associated polypeptides in mitochondria of Saccharomyces cerevisiae. Methods Enzymol. 260: 495-503.

Lazowska, J., M. Claisse, A. Gargouri, Z. Kotylak, A. Spyridakis, and P.P. Slonimski. 1989. Protein encoded by the third intron of cytochrome $b$ gene in Saccharomyces cerevisiae is an mRN A maturase. Analysis of mitochondrial mutants, RN A transcripts, proteins, and evolutionary relationships. J. Mol. Biol. 205: 275-289.

Leonhard, K., J.M. Herrmann, R.A. Stuart, G. Mannhaupt, W. N eupert, and T. Langer. 1996. AAA proteases with catalytic sites on opposite membrane surfaces comprise a proteolytic system for the ATP-dependent degradation of inner membrane proteins in mitochondria. EMBO J. 15: 4218-4229.

Lewin, A.S., J. Thomas Jr., and H.K. Tirupati. 1995. Cotranscriptional splicing of a group I intron is facilitated by the Cbp2 protein. Mol. Cell. Biol. 15: 6971-6978.

Manthey, G.M. and J.E. McEwen. 1995. The product of the nuclear gene PET309 is required for translation of mature mRNA and stability or production of intron-containing RNAs derived from the mitochondrial COX1 locus of Saccharomyces cerevisiae. EMBO J. 14: 4031-4043.

Maurizi, M.R. 1992. Proteases and protein degradation in Escherichia coli. Experientia 48: 178-201.

M cEwen, J.E., C. Ko, B. Kloeckner-Gruissem, and R.O. Poyton. 1986. N uclear functions required for cytochrome c oxidase biogenesis in Saccharomyces cerevisiae. Characterization of mutants of 34 complementation groups. J. Biol. Chem. 261: 11872-11879.

McKee, E.E. and P. Poyton. 1984. Mitochondrial gene expression in Saccharomyces cerevisiae. Optimal conditions for protein synthesis in isolated mitochondria. J. Biol. Chem. 259: 9320-9331.

Mulero, J.J. and T.D. Fox. 1993. PET111 acts in the $5^{\prime}$-leader of Saccharomyces cerevisiae mitochondrial COX2 mRNA to promote its translation. Genetics 133: 509-516.

Muroff, I. and A. Tzagoloff. 1990. CBP7 codes for a co-factor 
required in conjunction with a mitochondrial maturase for splicing of its cognate intervening sequence. EMBO J. 9: 2765-2773.

N obrega, F.G. and A. Tzagoloff. 1980. Assembly of the mitochondrial membrane system. DNA sequence and organization of the cytochrome $b$ gene in Saccharomyces cerevisiae. J. Biol. Chem. 255: 9828-9837.

Panzner, S., L. Dreier, E. Hartmann, S. Kostka, and T. Rapoport. 1995. Posttranslational protein transport in yeast reconstituted with a purified complex of Sec proteins and Kar2p. Cell 81: 561-570.

Pel, H.J. and L.A. Grivell. 1993. The biology of yeast mitochondrial introns. Mol. Biol. Rep. 18: 1-13.

-_- 1994. Protein synthesis in mitochondria. Mol. Biol. Rep. 19: 183-194.

Perlman, P.S. 1990. Genetic analysis of RNA splicing in yeast mitochondria. Methods Enzymol. 181: 539-558.

Rep, M. and L.A. Grivell. 1996. The role of protein degradation in mitochondrial function and biogenesis. Curr. Genet. 30: 367-380.

Rep, M., J. N ooy, E. Guélin, and L.A. Grivell. 1996a. Three genes for mitochondrial proteins suppress null-mutations in both AFG3 and RCA1 when overexpressed. Curr. Genet. 30: 206-211.

Rep, M., M. van Dijl, K. Suda, G. Schatz, L.A. Grivel I, and C.K. Suzuki. 1996b. Promotion of mitochondrial membrane complex assembly by a proteolytically inactive yeast Lon. Science 274: 103-106.

Rödel, G. 1997. Translational activator proteins required for cytochrome b synthesis in Saccharomyces cerevisiae. Curr. Genet. 31: 375-379.

Sambrook, J., E.F. Fritsch, and T. Maniatis. 1989. Molecular cloning: A laboratory manual. Cold Spring Harbor Laboratory Press, Cold Spring Harbor, NY.

Schmitt, M.E., T.A. Brown, and B.L. Trumpower. 1990. A rapid and simple method for preparation of RNA from Saccharomyces cerevisiae. Nucleic Acids Res. 18: 3091-3092.

Seraphin, B., A. Boulet, M. Simon, and G. Faye. 1987. Construction of a yeast strain devoid of mitochondrial introns and its use to screen nuclear genes involved in mitochondrial splicing. Proc. Natl. Acad. Sci. 84: 6810-6814.

Sherman, F. 1991. Getting started with yeast. Methods Enzymol. 194: 3-21.

Sikorski, R.S. and P. Hieter. 1989. A system of shuttle vectors and yeast host strains designed for efficient manipulation of DN A in Saccharomyces cerevisiae. Genetics 122: 19-27.

Stirling, C.J. and E.W. Hewitt. 1992. The S. cerevisiae SEC65 gene encodes a component of yeast signal recognition particle with homology to human SRP19. Nature 356: 534-537.

Suzuki, C.K., K. Suda, N. Wang, and G. Schatz. 1994. Requirement for the yeast gene LON in intramitochondrial proteolysis and maintenance of respiration. Science 264: 273-276.

Suzuki, C.K., M. Rep, J.M. van Dijl, K. Suda, L.A. Grivell, and G. Schatz. 1997. ATP-dependent proteases that al so chaperone protein biogenesis. Trends Biochem. Sci. 22: 118-123.

Tauer, R., G. Mannhaupt, R. Schnall, A. Pajic, T. Langer, and H. Feldmann. 1994. Y ta10p, a member of a novel ATPase family in yeast, is essential for mitochondrial function. FEBS Lett. 353: 197-200.

Teichmann, U., L. van Dyck, B. Guiard, H. Fischer, R. Glockshuber, W. Neupert, and T. Langer. 1996. Substitution of PIM 1 protease in mitochondria by Escherichia coli Lon protease. J. Biol. Chem. 271: 10137-10142.

Thorsness, P.E., K.H. White, and T.D. Fox. 1993. Inactivation of YME1, a member of the ftsH-SEC 18-PAS1-CDC 48 family of putative ATPase-encoding genes, causes increased escape of
DNA from mitochondria in Saccharomyces cerevisiae. Mol. Cell. Biol. 13: 5418-5426.

Tzagol off, A. and A.M. M yers. 1986. Genetics of mitochondrial biogenesis. Annu. Rev. Biochem. 55: 249-285.

Tzagol off, A., J. Yue, J. Jang, and M.F. Paul. 1994. A new member of a family of ATPases is essential for assembly of mitochondrial respiratory chain and ATP synthetase complexes in Saccharomyces cerevisiae. J. Biol. Chem. 269: 2614426151.

van Dyck, L., D.A. Pearce, and F. Sherman. 1994. PIM 1 encodes a mitochondrial ATP-dependent protease that is required for mitochondrial function in the yeast Saccharomyces cerevisiae. J. Biol. Chem. 269: 238-242.

Wagner, I., H. Arlt, L. van Dyck, T. Langer, and W. Neupert. 1994. Molecular chaperones cooperate with PIM 1 protease in the degradation of misfolded proteins in mitochondria. EMBO J. 13: 5135-5145.

Wagner, I., L. van Dyck, A. Savel'ev, W. N eupert, and T. Langer. 1997. Autocatalytic processing of the ATP-dependent PIM 1 protease: Crucial function of a pro-region for sorting to $\mathrm{mi}$ tochondria. EMBO J. 16: 7317-7325.

Yaffe, M.P. and G. Schatz. 1984. Two nuclear mutations that block mitochondrial protein import in yeast. Proc. Natl. Acad. Sci. 81: 4819-4823.

Zinser, E. and G. Daum. 1995. Isolation and biochemical characterization of organelles from the yeast Saccharomyces cerevisiae. Yeast 11: 493-536. 


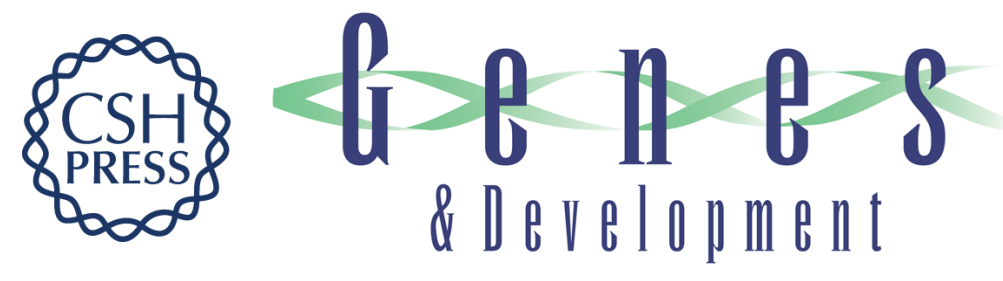

\section{The ATP-dependent PIM1 protease is required for the expression of intron-containing genes in mitochondria}

Luc van Dyck, Walter Neupert and Thomas Langer

Genes Dev. 1998, 12:

Access the most recent version at doi:10.1101/gad.12.10.1515

References This article cites 70 articles, 24 of which can be accessed free at: http://genesdev.cshlp.org/content/12/10/1515.full.html\#ref-list-1

License

Email Alerting

Receive free email alerts when new articles cite this article - sign up in the box at the top Service right corner of the article or click here.

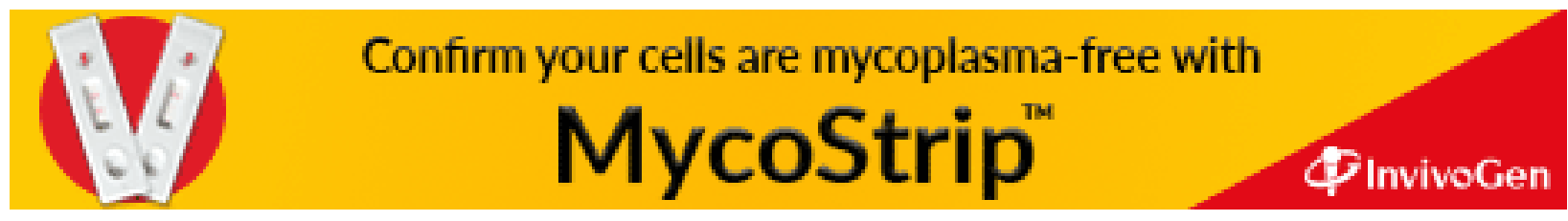

